\title{
Structure and Expression of $c$-rel, the Cellular Homolog to the Oncogene of Reticuloendotheliosis Virus Strain T
}

\author{
IRVIN S. Y. CHEN, † KIRK C. WILHELMSEN, AND HOWARD M. TEMIN* \\ McArdle Laboratory for Cancer Research, University of Wisconsin, Madison, Wisconsin 53706
}

Received 21 June 1982/Accepted 27 August 1982

\begin{abstract}
The cellular homolog of the onc sequences in the avian retrovirus reticuloendotheliosis virus strain $\mathrm{T}(v-r e l)$ was studied by molecular cloning and nucleic acid hybridization. In contrast to $v$-rel sequences, which are 1.4 kilobase pairs long, the cellular homolog, $c$-rel, from line 15B chickens is at least 25 kilobase pairs long, with multiple apparent introns. A 4.0-kilobase polyadenylic acid-containing RNA transcript is the primary species of $c$-rel RNA present in uninfected chicken cells. Sequences at the $3^{\prime}$ end of this $c$-rel RNA are not present in $v$-rel.
\end{abstract}

Reticuloendotheliosis virus strain T (REV-T) is a highly oncogenic avian retrovirus that causes rapidly fatal leukemia in newly hatched chicks and turkey poults (27). Like the genomes of other highly oncogenic avian retroviruses, the genome of REV-T contains sequences ( $v$-rel) that are not necessary for viral replication $(6,7$, $13,14)$. The $v$-rel sequences are necessary for the transforming properties of REV-T (7a). These sequences are related to sequences in the genomes of uninfected chickens and turkeys (crel) $(7,31)$. The $c$-rel sequences in turkeys are believed to be the progenitors of the v-rel sequences.

Previous studies have indicated that the cellular homolog of $v$-rel is arranged differently than $v$-rel of REV-T (7). Multiple large DNA fragments having homology to $v$-rel have been detected in cellular DNA, indicating either that $c$-rel is distributed over a large region of cellular DNA or that $c$-rel represents a small gene family. These previous studies were extended by obtaining molecular clones of $c$-rel from chicken DNA and comparing the structure of $c$-rel with the structure of $v$-rel. Our results show that, rather than being duplicated, $c$-rel is distributed over a large contiguous region of chicken DNA. In addition, a comparison of c-rel mRNA and $v$-rel DNA showed that at least part of a 3' c-rel exon is not present in $v$-rel. (See Addendum in Proof.)

\section{MATERIALS AND METHODS}

Cells. The general sources and procedures for obtaining and propagating avian fibroblastic cells have been described previously (12).

$\dagger$ Present address: Division of Hematology and Oncology, School of Medicine, University of California at Los Angeles, Los Angeles, CA 90024.
Sources of DNAs. Line 15B chicken DNA was extracted from line 15B chicken embryo fibroblasts as previously described (7).

Clone 2-20-4 is a variant REV-T proviral clone in Charon 4A (7).

pREV-T 3 is a circularly permuted molecular clone of REV-T unintegrated circular DNA in pBR322 (7a). Infectious virus that can transform chicken spleen cells in culture can be recovered from this DNA.

Ch4A REV-T 52B is a molecular clone of infectious REV-T proviral DNA (Wilhelmsen, unpublished data).

Clones propagated in pBR322 were grown in Escherichia coli strain HB101 as previously described (24). Clones propagated in the bacteriophage vectors Charon $4 A(5)$ and $\lambda 1059$ (16) were grown in $E$. coli strains DP50 supF and C600, respectively.

Source of RNA. Chicken spleens were obtained from 2- to 3-week-old White Leghorn $\times$ New Hampshire Red chickens (University of Wisconsin, Madison). The spleens were stored at $-70^{\circ} \mathrm{C}$. For extraction of RNA, the spleens were ground to a fine powder with a mortar and pestle over dry ice. The tissue was lysed rapidly with urea lysis buffer (15), and total cellular RNA was purified by centrifugation in $\mathrm{CsCl}$ (22). Polyadenylic acid containing RNA was purified by using oligodeoxythymidylic acid cellulose (2).

Preparation of molecular clones. A recombinant DNA library in Charon 4A constructed from a partial EcoRI digest of line 15B chicken DNA (generously supplied by Ed Fritsch, Michigan State University, East Lansing), was screened by hybridization to the middle $v$-rel probe (see Fig. 1). Several positive clones were obtained. A preliminary analysis showed that all of the clones were identical except for the orientation of the chicken DNA insertion with respect to Charon $4 \mathrm{~A}$; therefore, only one of these clones, $\lambda \mathrm{Ch} 4 \mathrm{~A} c$-rel 1 , was characterized in detail.

A recombinant DNA library of line $15 B$ chicken DNA in $\lambda 1059$ was constructed by using BamHI chicken DNA fragments selected on an $\mathrm{NaCl}$ gradient to be between 10 and 25 kilobase pairs (kbp) long. The $\lambda 1059$ c-rel 3 and $\lambda 1059$ c-rel 4 recombinant clones 
were obtained by using DNA fragments derived from the $c$-rel insertion in $\lambda \mathrm{Ch} 4 \mathrm{~A} c$-rel 1 as hybridization probes. The HindIII DNA fragment at 16.3 to $18.9 \mathrm{kbp}$ and the HindIII-EcoRI DNA fragment at 21.6 to 23.6 kbp were subcloned from $\lambda \mathrm{Ch} 4 \mathrm{~A}$ c-rel 1 into pBR322 (see Fig. 2). The chicken DNA insertions were purified from pBR322 DNA and used as hybridization probes.

Another recombinant DNA library of line 15B chicken DNA was constructed in $\lambda 1059$ by using $B$ glII DNA fragments selected on an $\mathrm{NaCl}$ gradient to be between 9.0 and $20 \mathrm{kbp}$ long. The $\lambda 1059$ c-rel 5 recombinant clone was obtained by using the BamHIEcoRI DNA fragment (4.5 to $6.5 \mathrm{kbp}$ [see Fig. 2]) subcloned from $\lambda 1059$ c-rel 4 into pBR322 as a hybridization probe to screen this library.

Subclones of the $c$-rel clones in pBR322 were prepared as previously described (24).

Gel electrophoresis, nucleic acid transfer to nitrocellulose, and molecular hybridization. Gel electrophoresis, transfer to nitrocellulose, and molecular hybridization of DNA have been described previously (7). The relative amounts of hybridization were determined by densitometry tracing of autoradiograms with a JoyceLoebl microdensitometer.

RNA was denatured with glyoxal, subjected to electrophoresis, and transferred to nitrocellulose as described by Thomas (28). Molecular size markers were visualized by soaking the gel in a solution containing $0.02 \%$ methylene blue, $0.02 \%$ sodium acetate, and $0.02 \%$ acetic acid. RNA treated in this manner does not hybridize efficiently. Therefore, the lanes containing molecular size markers were cut from the remainder of the gel.

The RNA immobilized on a filter was hybridized at $42^{\circ} \mathrm{C}$ for 24 to $36 \mathrm{~h}$ in a solution containing $50 \%$ deionized formamide, $5 \times \mathrm{SSC}(1 \times \mathrm{SSC}$ is $0.15 \mathrm{M}$ $\mathrm{NaCl}$ plus $0.015 \mathrm{M}$ sodium citrate, $\mathrm{pH} 7.0$ ), $1 \times$ Denhardt solution, $0.1 \%$ sodium dodecyl sulfate, and $50 \mu \mathrm{g}$ of sonicated salmon sperm DNA per $\mathrm{ml}(28)$.

Hybridization probes ([ $\left.{ }^{32} \mathrm{P}\right] \mathrm{DNA}$ or $\left.\left[{ }^{32} \mathrm{P}\right] \mathrm{cDNA}\right)$ were prepared as previously described (7).

\section{RESULTS}

Previous experiments in which a limited region of $v$-rel was used as a probe indicated that $c$-rel in different avian species is structurally more complex than v-rel in REV-T (7); that is, there is either a single complex $c$-rel gene or a $c$-rel gene family in chicken DNA. To differentiate between these two possibilities, a series of DNA subclones that included all $1.4 \mathrm{kbp}$ of $v$-rel were made from different regions of REV-T and were used as hybridization probes in the experiments described below (Fig. 1). (The sequences in the $3^{\prime} v$-rel probe related to the helper virus, reticuloendotheliosis virus strain $\mathrm{A}$, do not hybridize to uninfected chicken DNA [unpublished data].)

Kestriction enzyme analysis of $c$-rel in chicken DNA. The $v$-rel hybridization probes hybridized strongly to several DNA fragments in chicken DNA digested with either EcoRI, BamHI, or HindIII. After EcoRI digestion of chicken DNA,
5.5- and 1.9-kbp DNA fragments hybridized with the 5'v-rel (HindIII-EcoRI) probe, 21.0and 9.0-kbp fragments hybridized with the middle $v$-rel probe, and 10.0- and 9.0-kbp fragments hybridized with the $3^{\prime} v$-rel probe (Fig. 1). With the exception of the 9.0-kbp DNA fragment, the DNA fragments detected by the different $v$-rel probes were different in size. The combined size of the five different DNA fragments was approximately $47 \mathrm{kbp}$. Similarly, BamHI digestion of chicken DNA revealed 9.7- and 3.1-kbp c-rel DNA fragments with the 5 ' $v$-rel probe, 21.0and 12.0-kbp fragments with the middle v-rel probe, and 21.0- and 5.0-kbp fragments with the $3^{\prime} v$-rel probe (Fig. 1). As described above for EcoRI digestion, five DNA fragments of different sizes were detected. The combined size of these fragments was approximately $50 \mathrm{kbp}$. HindIII digestion of chicken DNA also revealed the following $c$-rel DNA fragments: 4.0- and 0.5kbp fragments with 5'v-rel; 6.5-, 2.5-, 1.7-, and 1.3-kbp fragments with middle $v$-rel; and 12.0and 6.5-kbp fragments with $3^{\prime} v$-rel (Fig. 1). Six of the DNA fragments were different in size. The combined size of these fragments was approximately $28 \mathrm{kbp}$.

With the exceptions of the 9.0-kbp EcoRI DNA fragment, the 21.0-kbp BamHI DNA fragment, and the 6.5-kbp HindIII DNA fragment, none of the DNA fragments generated by digestion with the three enzymes hybridized with more than one of the nonoverlapping $v$-rel probes. Therefore, each different c-rel DNA fragment could not represent a different member of a c-rel gene family. Thus, these results indicate either that at least five regions of homology io $v$-rel are distributed over approximately 28 to $50 \mathrm{kbp}$ or more of chicken DNA or, alternatively, that different parts of $v$-rel are homologous to unlinked regions in chicken DNA.

Molecular cloning of $c$-rel (chicken). To determine more precisely the arrangement of the regions in chicken DNA homologous to v-rel, molecular clones of $c$-rel were obtained from chicken DNA. Four lambda recombinant clones were obtained by plaque hybridization from recombinant DNA libraries of line $15 B$ chicken DNA (see above). Detailed restriction endonuclease cleavage maps of the chicken DNA insertions were made and compared with each other (Fig. 2). A total of $\mathbf{4 0} \mathrm{kbp}$ of contiguous chicken DNA was cloned in overlapping DNA fragments of the four recombinant lambda clones.

Several lines of evidence indicate that the recombinant clones did not suffer gross alter ations as a result of the cloning process. First, the regions of overlap between the clones were identical with respect to the positions of the restriction enzyme cleavage sites. Second, subclones were constructed from various regions of 


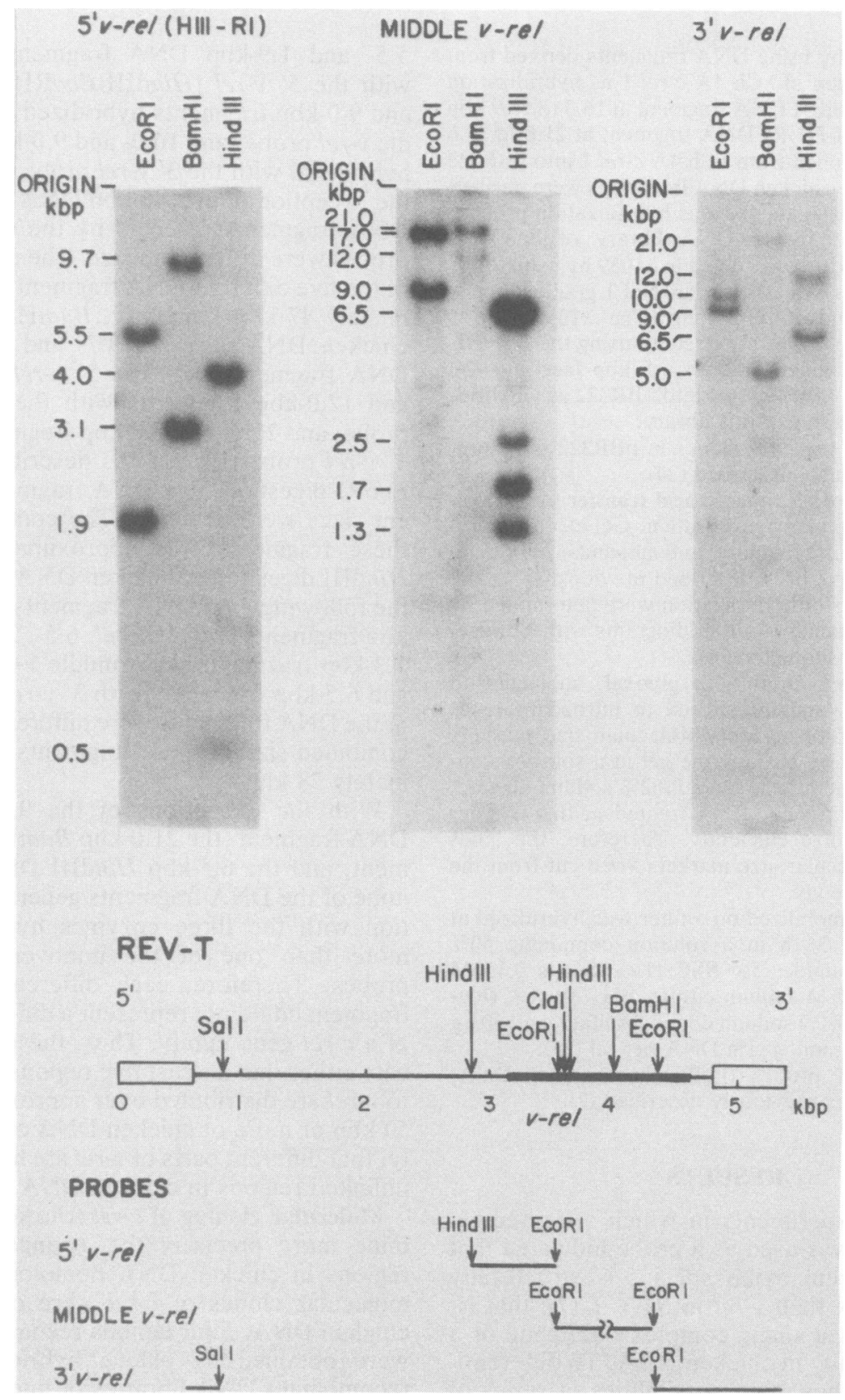

FIG. 1. Hybridization of $v$-rel probes to line 15B chicken cellular DNA. Chicken DNA was prepared as described in the text. DNA $(20 \mu \mathrm{g})$ was digested with either EcoRI, BamHI, or HindIII and was subjected to electrophoresis in a $0.8 \%$ agarose gel. Chicken sequences homologous to each of the indicated $v$-rel probes were detected by hybridization as described in the text. The size of each of the DNA fragments is shown to the left of each autoradiogram. The positions of the restriction enzyme cleavage sites in REV-T DNA are shown below the autoradiograms. Only the BamHI site within $v$-rel is indicated. $5^{\prime}$ and $3^{\prime}$ refer to the orientation of the viral DNA with respect to the viral RNA. The numbers indicate the distances from the $5^{\prime}$ end of viral DNA. The sources of the hybridization probes from REV-T DNA are indicated by the bars below the map of REV-T. The hybridization probes were made from subcloned DNA fragments of REV-T which were purified free from the plasmid vector before radioactive labeling. The sources of the REV-T DNA in each of the subclones were as follows: 5' v-rel (HindIII-EcoRI), infectious REV-T proviral recombinant clone (Charon 4A REV-T 52B) (Wilhelmsen, unpublished data); middle $v$-rel, variant REV-T proviral clone 2-20-4 (7); 3'v-rel, infectious REV-T unintegrated circular DNA clone (pREV-T 3) (7a). The break in the middle $v$-rel probe indicates a deletion of about 50 base pairs (7). 
the clones and were used as hybridization probes with restriction enzyme-digested chicken DNA. In all cases, except when the DNA contained highly repetitive sequences (see below), the subclones hybridized to a DNA fragment in restriction enzyme-digested chicken DNA of the same size as the subcloned DNA fragment (data not shown). Third, cloned DNA fragments that contained regions homologous to $v$-rel were identical in size to the fragments detected by $v$-rel probes in chicken DNA (see below). Therefore, the $c$-rel clones represent an accurate copy of the $c$-rel region of chicken DNA.

The positions of regions of the clones having homology to $v$-rel were determined by hybridization, using $\left[{ }^{32} \mathrm{P}\right] \mathrm{cDNA}$ made to REV-T RNA. Seven regions of homology were located in the following fragments: a $B g l I I-E c o R I$ DNA fragment at -1.0 to $1.0 \mathrm{kbp}$, a HindIII DNA fragment at 1.5 to $1.7 \mathrm{kbp}$, an EcoRI-HindIII DNA fragment at 6.5 to $6.9 \mathrm{kbp}$, a ClaI-HindIII DNA fragment at 18.7 to $19.0 \mathrm{kbp}$, a HindIII-ClaI DNA fragment at 19.0 to $20.6 \mathrm{kbp}$; a BglIIEcoRI DNA fragment at 22.6 to $23.6 \mathrm{kbp}$, and an EcoRI-HindIII DNA fragment at 23.5 to $28 \mathrm{kbp}$ (Fig. 2). (See Addendum in Proof.) These results were confirmed by an electron microscopic heteroduplex analysis (Wilhelmsen, manuscript in preparation).

A comparison of the c-rel DNA fragments detected by hybridization of $v$-rel to chicken DNA (Fig. 1) and the cloned region of c-rel DNA indicated that the majority of the c-rel DNA fragments detected in cellular DNA were present in the clones. All of the fragments detected by the $5^{\prime} v$-rel (HindIII-EcoRI) and middle v-rel probes were present in the cloned region of $c$ rel. However, not all of the fragments detected by the 3' $v$-rel probe were present in the cloned region of c-rel. Among the DNA fragments detected by the 3' $v$-rel probe in chicken cellular DNA (Fig. 1), the 9.0-kbp EcoRI DNA fragment, the 21.0-kbp BamHI DNA fragment, and the 6.5-kbp HindIII DNA fragment were present in the cloned region corresponding to the rightmost region of homology in c-rel. The other DNA fragments detected in chicken cellular DNA (a 10.0-kbp EcoRI DNA fragment, a 5.0kbp BamHI DNA fragment, and a 12.0-kbp HindIII DNA fragment) were not present in the cloned region of $c$-rel. Hybridization to these fragments was relatively weak, but reproducible.

Experiments designed to determine the locations or origins of these additional regions of $c$-rel were unsuccessful. These regions did not hybridize to the helper virus-related sequences present in the $3^{\prime} v$-rel probe (Fig. 1 shows the source of the 3' $v$-rel probe [data not shown]). Therefore, these sequences are rel specific. These sequences may be linked to the cloned c-rel region, in which case they would be separated from the other regions of homology by at least 9 and $12 \mathrm{kbp}$ at the left and right, respectively. Alternatively, they are not linked or represent weak homology to sequences distantly related to 3' v-rel.

Only one EcoRI fragment in turkey DNA was detected with the $3^{\prime} v$-rel probe, and the region homologous to $v$-rel in this turkey DNA fragment (which has been cloned) was conserved between chicken and turkey DNAs (Wilhelmsen, manuscript in preparation). This result most likely indicates that the 10-kbp EcoRI fragment in chicken DNA that was detected with the $3^{\prime}$ $v$-rel probe is not linked to c-rel and is only distantly related to the $3^{\prime} v$-rel sequences.

Thus, these results demonstrate that the majority of the regions of $c$-rel that hybridize to $v$-rel are arranged contiguously in a single region of the chicken genome. The total size of this c-rel region is about $25 \mathrm{kbp}$. Seven regions separable by restriction enzyme digestion are homologous to $v$-rel. These seven regions of homology are separated by DNA sequences ranging in size from about 2 to $12 \mathrm{kbp}$. A DNA electron microscopic heteroduplex analysis indicated that the regions of homology at 23.0 and $26.0 \mathrm{kbp}$ are not contiguous (Wilhelmsen, manuscript in preparation). The regions of homology separated by a single restriction enzyme cleavage site at 18.8 to $19.1 \mathrm{kbp}$ may be contiguous Therefore, $c$-rel has a minimum of six discontinuous regions of homology to $v$-rel.

Although the organization of c-rel is quite different from that of $v$-rel, the regions of $c$-rel homologous to $v$-rel appear to be arranged in the same orientation as the corresponding regions of $v$-rel; that is, $5^{\prime} v$-rel, middle $v$-rel, and $3^{\prime} v$-rel hybridize to DNA fragments from the left, middle, and right of c-rel, respectively (Fig. 2). (Note that $5^{\prime} v$-rel [HindIII-HindIII] contains approximately $0.1 \mathrm{kbp}$ more of $v$-rel to the $3^{\prime}$ side than $5^{\prime} v$-rel [HindIII-EcoRI]. This additional $0.1 \mathrm{kbp}$ hybridizes to the next two leftmost fragments of c-rel having homology to v-rel.) Therefore, the left side of $c$-rel was designated the $5^{\prime}$ end with respect to $v$-rel.

The single HindIII site in $v$-rel is located at 3.6 kbp in REV-T (Fig. 1). About $0.6 \mathrm{kbp}$ of $v$-rel is located $5^{\prime}$ to this HindIII site. This $0.6 \mathrm{kbp}$ of $v$-rel (the total size of $v$-rel is $1.4 \mathrm{kbp}$ ) is homologous to four discontinuous regions in $c$-rel located $5^{\prime}$ to the HindIII site at $19.0 \mathrm{kbp}$ (Fig. 2, homology to 5 'v-rel [HindIII-HindIII]). Therefore, the average size of these homologous regions in c-rel is less than $0.2 \mathrm{kbp}$. (This size was confirmed by an electron microscopic heteroduplex analysis [Wilhelmsen, manuscript in preparation].) Two of these regions of homology, at 7.0 and $19.0 \mathrm{kbp}$, are separated by a large region of chicken DNA about $12.0 \mathrm{kbp}$ long. 


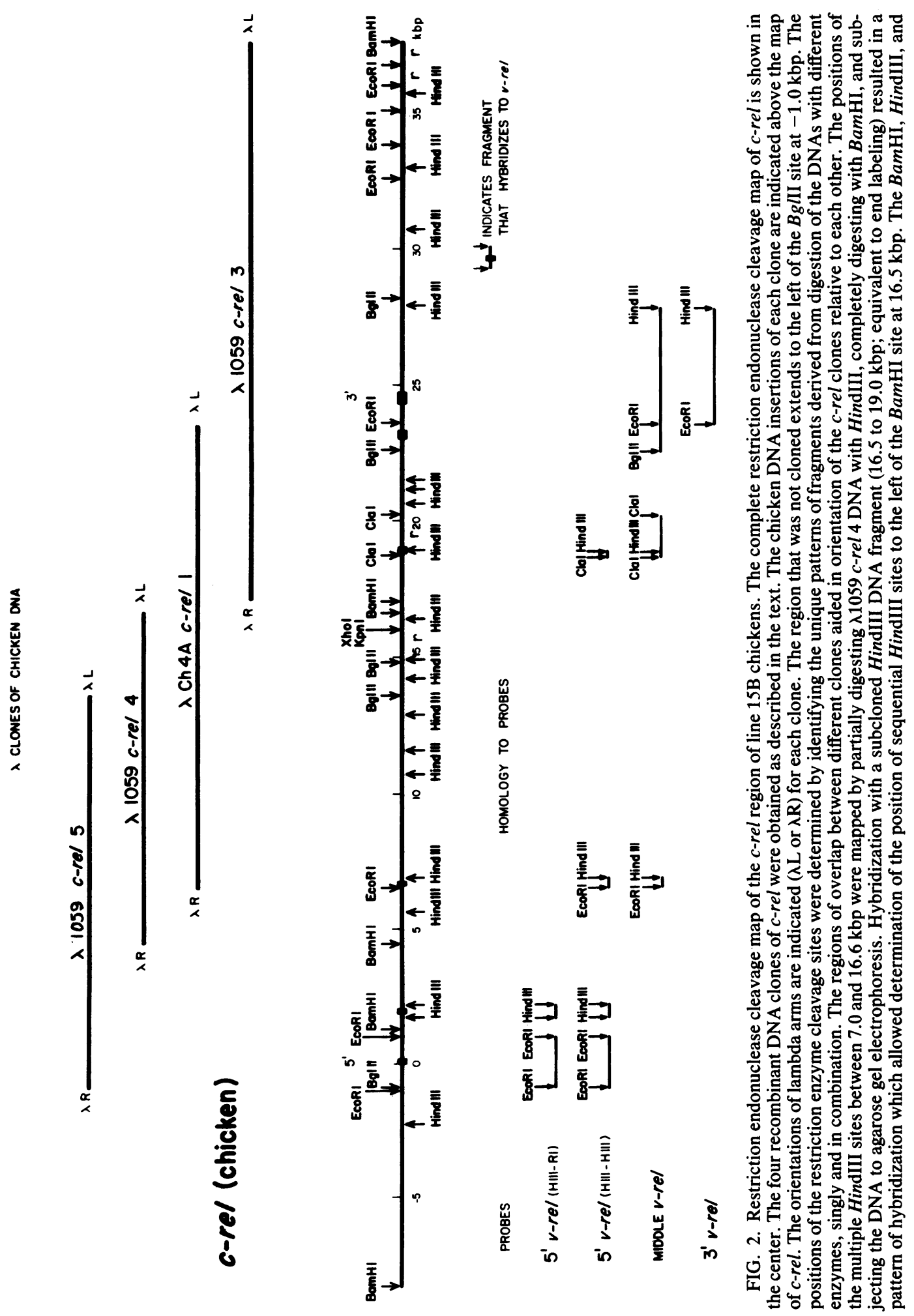




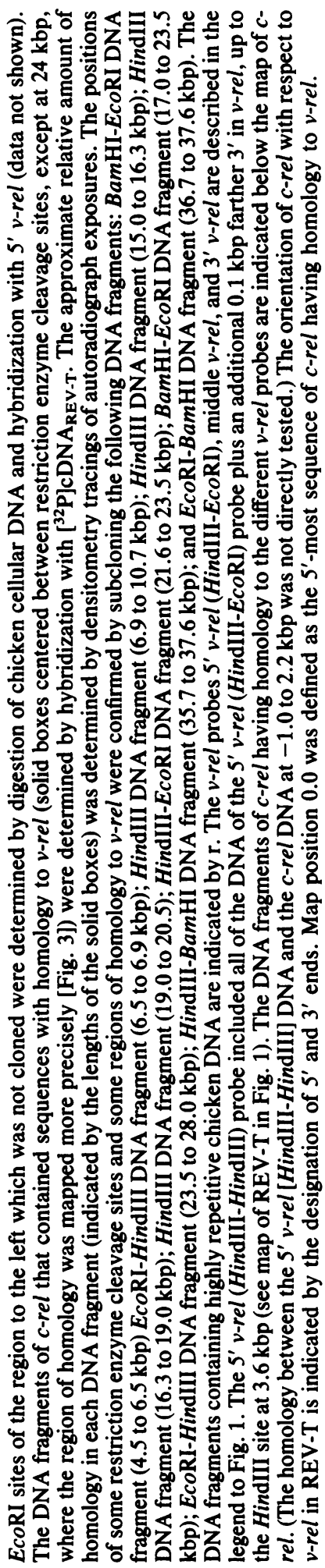

A total of $0.8 \mathrm{kbp}$ of $v$-rel is present $3^{\prime}$ to the HindIII site in v-rel. The sequences in this region, which comprises more than one-half of $v$-rel, are homologous to regions within the 3' one-fifth of $c$-rel. Most of the homology is present within two regions of $c$-rel, which are located at 23.0 and $24.5 \mathrm{kbp}$. A DNA electron microscopic heteroduplex analysis confirmed this arrangement of homologous regions (Wilhelmsen, manuscript in preparation).

c-rel DNA fragments in the cloned region containing highly repetitive chicken DNA sequences were determined by hybridization, using radioactively labeled chicken DNA. Four repetitive regions were located at about 15.0, $20.0,36.0$, and $38.0 \mathrm{kbp}$ (Fig. 2, regions r).

Expression of $c$-rel in chicken spleen cells. The structure of $c$-rel resembles that of a cellular gene having exons and introns. The large regions of $c$-rel that separate the regions homologous to $v$-rel may be intervening sequences with respect to $c$-rel mRNA. In that case, $c$-rel mRNA would be colinear to $v$-rel sequences in REV-T. Alternatively, the regions of homology to $v$-rel may differ from the exons for $c$-rel. To determine whether any of the regions in $c$-rel DNA that are not homologous to $v$-rel DNA are present in $c$ rel mRNA, RNA preparations from chicken cells were hybridized with DNA probes from different regions of cloned $v$-rel and c-rel.

Preliminary experiments showed that the levels of RNA homologous to v-rel in chicken hemopoietic tissue were two- to threefold higher than the levels in muscle or fibroblasts (data not shown). Levels of RNA similar to the level in hemopoietic tissue were present in a Marek's virus-transformed cell line, RP-4 (30), and a lymphoid leukosis cell line, RP-13 (30). A 4.0kilobase (kb) polyadenylic acid-containing RNA species was detected in chicken spleen cells by using the $5^{\prime} v$-rel and middle $v$-rel hybridization probes (Fig. 3). The 3'v-rel hybridization probe did not detect any RNA species, possibly because of the small region (less than 250 base pairs) of $v$-rel in this probe. Occasionally, in some RNA preparations a larger species $(6.0 \mathrm{~kb})$ was detected in addition to the 4.0-kb species. However, the 4.0-kb species was the only species consistently detected in several different preparations of RNA. Presumably, the 4.0-kb species is the mRNA for $c$-rel. The $6.0-\mathrm{kb}$ species may be a precursor RNA.

Since the $v$-rel region in REV-T is $1.4 \mathrm{kbp}$ long, it is apparent that the combined size of $c$-rel exons (about $4.0 \mathrm{kbp}$, as determined from the size of $c$-rel mRNA) is about $2.6 \mathrm{kbp}$ larger than $v$-rel. To determine the positions of the exons in $c$-rel DNA that are not represented in $v$ rel, chicken polyadenylic acid-containing RNA was hybridized with DNA probes from dif- 


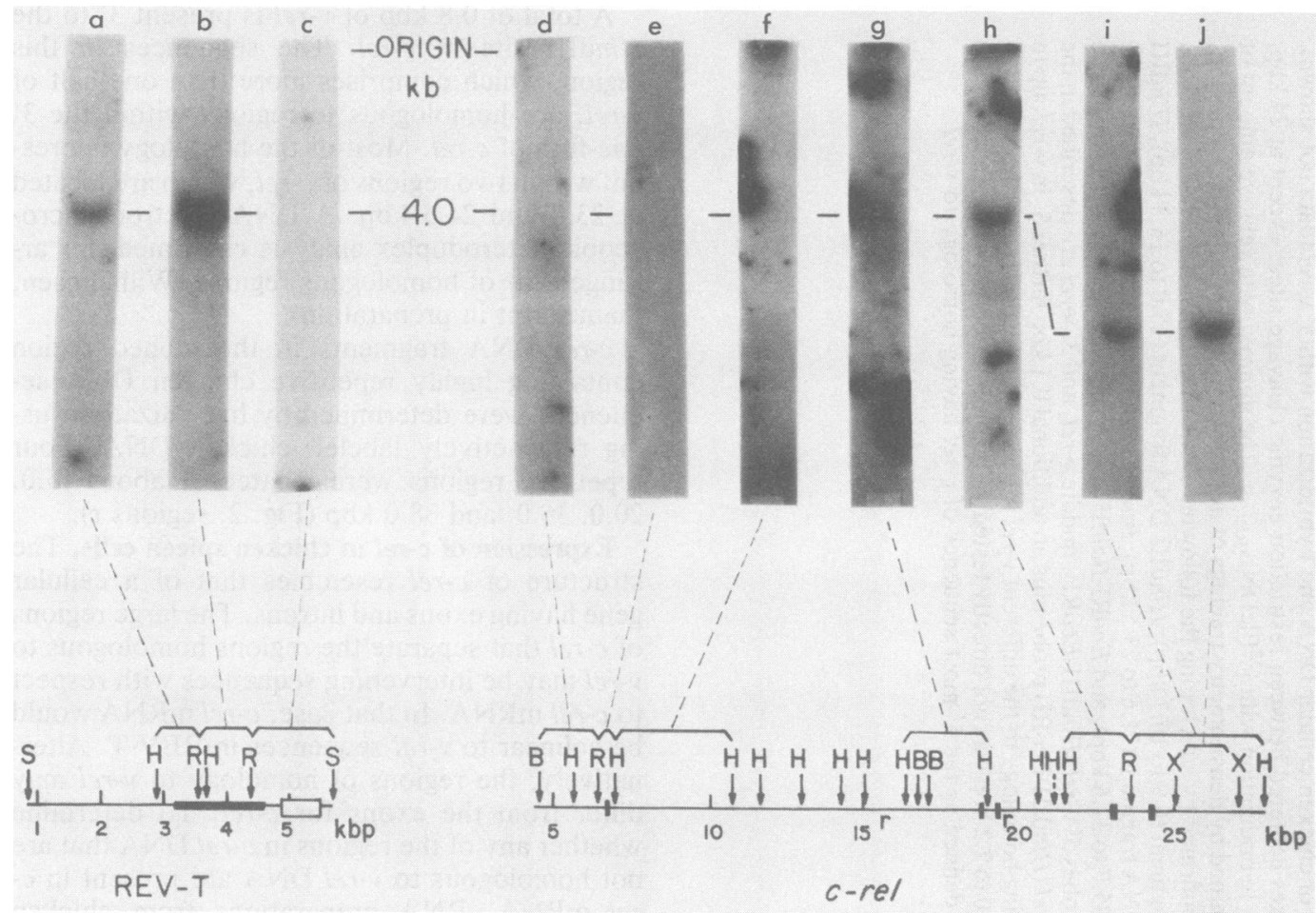

FIG. 3. Hybridization of $v$-rel and $c$-rel DNA fragments to chicken spleen polyadenylic acid-containing RNA. Polyadenylic acid-containing RNA was prepared from chicken spleen cells as described in the text. RNA (25 $\mu \mathrm{g})$ was subjected to gel electrophoresis in parallel lanes (except lanes $\mathrm{i}$ and $\mathrm{j}$, which were from a separate gel). Specific RNA species were detected by hybridization with the specific DNA probes indicated. The source of the DNA probe for each lane is indicated on the restriction enzyme cleavage site maps of REV-T and $c$-rel below the autoradiographs. The three $v$-rel probes correspond to the $5^{\prime} v$-rel (HindIII-EcoRI), middle $v$-rel, and $3^{\prime} v$-rel probes described in the legend to Fig. 1. The map of REV-T is shown as a permuted sequence about the SalI site that is $\mathbf{0 . 8 6} \mathbf{~ k b p}$ from the $5^{\prime}$ end of viral DNA (Fig. 1). Only part of the cloned region of $c$-rel is shown in the map of $c$-rel. The positions of the $\mathrm{XbaI}$ sites were determined only for the EcoRI-HindIII DNA fragment at 23.5 to $28.5 \mathrm{kbp} . \mathrm{r}$ indicates the presence of repetitive chicken DNA. S, SalI; H, HindIII; R, EcoRI; B, BamHI; X, XbaI.

ferent regions of c-rel (Fig. 3). Some of these DNA probes contained sequences homologous to $v$-rel. Others were entirely nonhomologous to $v$-rel.

No detectable hybridization to RNA was observed with any of the $c$-rel DNA fragments that were from 5.0 to $10.7 \mathrm{kbp}$ on the $c$-rel map (Fig. $3)$. Neither fragments which contained homology to v-rel nor fragments from apparent intervening regions hybridized. This result indicates that regions of homology to c-rel mRNA, if present at all, are too small to give detectable hybridization. (The lack of hybridization obtained with fragments having homology to v-rel might have been a result of the small size of the regions of homology to $v$-rel.)

The c-rel HindIII-HindIII, HindIII-EcoRI, and EcoRI-HindIII DNA fragments at 16.3 to $19.0,21.6$ to 23.5 , and 23.5 to $28.0 \mathrm{kbp}$, respectively, hybridized to a 4.0-kb RNA species.
(DNA fragments at 15.0 to 16.3 and 19.0 to 20.5 kbp were not tested due to the presence of repetitive sequences.) These DNA fragments contained relatively large regions of homology to $v$-rel. Therefore, the hybridization to c-rel mRNA could be attributed to this region of homology. However, the intensity of hybridization with the EcoRI-HindIII DNA fragment (23.6 to $28.0 \mathrm{kbp})$ was much greater than expected based on the relative amount of homology to $v$-rel (data not shown), indicating possible homology between regions of this fragment not homologous to $v$-rel.

This possibility was tested by further dividing the EcoRI-HindIII DNA fragment into regions without homology to $v$-rel. Two $\mathrm{XbaI}$ sites $(25.0$ and $27.2 \mathrm{kbp}$ ) were mapped in this EcoRIHindIII DNA fragment. All of the homology to $v$-rel was located between the EcoRI site and the next $\mathrm{XbaI}$ site to the right (23.5 to $25.0 \mathrm{kbp}$ ) (Fig. 
3). Regions of $c$-rel farther to the right of the $X b a \mathrm{I}$ site at $25.0 \mathrm{kbp}$ did not hybridize to $v$-rel, delimiting the $3^{\prime}$ end of $v$-rel (data not shown). When the 25.0- to 27.2-kbp XbaI fragment was used for hybridization to chicken RNA, a 4.0-kb RNA species, which was the same size as the species detected by $v$-rel and other regions of $c$-rel, was detected (Fig. 3). Thus, this $3^{\prime}$ region of $c$-rel, although not present in v-rel, was transcribed into $c$-rel mRNA and, therefore, represents an exon of $c$-rel not present in $v$-rel.

\section{DISCUSSION}

Structural relationship between $c$-rel and $v$-rel. The structural relationship between $c$-rel in line $15 \mathrm{~B}$ chickens and $v$-rel in REV-T is similar to the relationships between most $v$-onc genes and their cellular c-onc homologs (8). $v$-rel is $1.4 \mathrm{kbp}$ long (Wilhelmsen, unpublished data), whereas in $c$-rel the regions of homology to $v$-rel span about $25 \mathrm{kbp}$ of chicken DNA. A minimum of six regions of homology to $v$-rel are separated by five apparent introns. The largest apparent intron is about $12.0 \mathrm{kbp}$ long.

Despite the dissimilarity in size between $c$-rel and $v$-rel, the regions in c-rel that are homologous to $v$-rel appear to be in the same order as in $v$-rel. Similar observations have also been made for the order of other $v$-onc genes and their respective $c$-onc counterparts $(3,9-11,18-21$, $23,26,29$ ).

In addition, there may be some restriction enzyme cleavage sites in common between $v$-rel and $c$-rel. The HindIII site in c-rel at $19.0 \mathrm{kbp}$ is likely to be conserved in $v$-rel at $3.6 \mathrm{kbp}$ (on the map of REV-T), since sequences $5^{\prime}$ and $3^{\prime}$ of the HindIII site in v-rel are homologous to sequences $5^{\prime}$ and $3^{\prime}$, respectively, of the HindIII site in c-rel. A DNA heteroduplex analysis is being performed to confirm this possibility. The EcoRI site at $6.5 \mathrm{kbp}$ and the ClaI site at $\mathbf{1 8 . 7}$ $\mathrm{kbp}$ in c-rel may also be present in $v$-rel, since these sites are present at analogous positions in the two sequences.

However, several enzyme sites in $v$-rel are not present in c-rel. The BamHI site at $4.1 \mathrm{kbp}$ and the EcoRI site at $4.3 \mathrm{kbp}$ (on the map of REV-T in Fig. 1) in v-rel are not present in c-rel, since c-rel contains no BamHI or EcoRI sites at the coresponding positions.

$v$-rel was probably derived from $c$-rel in turkeys $(7,31)$. Therefore, the restriction enzyme cleavage site differences between chicken c-rel and $v$-rel could represent differences between chicken $c$-rel and turkey $c$-rel. However, these enzyme sites in $v$-rel are also not present in c-rel from turkeys (Wilhelmsen and Temin, manuscript in preparation), indicating divergence between the $c$-rel and v-rel sequences rather than divergence of $c$-rel sequences in chickens and turkeys. Alternatively, the sites could have been created by the splicing of small exons. DNA sequencing will distinguish between these possibilities.

Most $c$-onc sequences appear to be conserved in the genomes of many vertebrate species that are widely divergent from the species from which the oncogenic virus originated. The organization of $c$-rel in turkeys is very similar to the organization described here for chickens with regard to the distribution and organization of regions of homology to $v$-rel (Wilhelmsen, manuscript in preparation). In addition, the structure of $c$-rel is also similar in other avian species (7). However, unlike most other $c$-onc sequences, $c$-rel appears not to be highly conserved in nonavian species $(7,31)$.

One particularly interesting relationship is that between $c$-rel and $c-a b l$, the cellular counterpart of the murine Abelson leukemia virus oncogene. There is no nucleic acid homology between $v$-abl and $v$-rel $(7,31)$, yet Abelson virus and REV-T cause lymphoid neoplasia in mice and chickens $(1,27)$, respectively, and the structures of the cellular genes are remarkably similar (3). Both genes are distributed over more than $20 \mathrm{kbp}$ of DNA, with multiple large apparent introns. In both genes, a major portion of the homology between the $v$-onc and c-onc sequences is located at the $3^{\prime}$ end. This similarity of structure between $c-a b l$ and $c$-rel and the similarity of function between $v$-abl and v-rel may indicate a common function for $c$-rel and $c$ $a b l$ in chickens and mice, respectively.

Expression of $c$-rel in chicken cells. RNA sequences homologous to $v$-rel are present at low levels in all chicken tissues which have been tested (data not shown). However, the levels of RNA are slightly higher (two- to threefold) in hemopoietic tissue than in other tissues. The significance of this observation is unclear, although it may be related to the postulated role of the expression of $c$-onc sequences in normal differentiation (4).

The mRNA of $c$-rel appears to be $4.0 \mathrm{~kb}$ long. This mRNA is about $2.6 \mathrm{~kb}$ larger than $v$-rel. We identified an exon at the $3^{\prime}$ end of $c$-rel which is not present in $v$-rel and may represent the DNA transcribed to give the additional $2.6 \mathrm{~kb}$. Many eucaryotic mRNAs have $3^{\prime}$ untranslated regions (17), and this region of $c$-rel may represent such a sequence. There also could be regions $5^{\prime}$ to the $c$-rel sequences which are transcribed. It is possible that these additional sequences are involved in the control of $c$-rel expression or, alternatively, they may encode additional peptides. The sequences $3^{\prime}$ to $c$-rel are also likely to contain signals for the termination of $c$-rel RNA transcription. Since termination signals prevent the formation of infectious virus (25), removal of 
such sequences was probably necessary for the formation of infectious REV-T. Experiments are in progress to determine whether these sequences have any effect on the transforming potential of REV-T by constructing a recombinant virus that contains $v$-rel plus the additional sequences from the $3^{\prime}$ end of $c$-rel.

The large regions in $c$-rel separating regions of homology to $v$-rel do not appear to be expressed as stable RNA, since we were unable to detect RNA transcripts by using DNA probes from parts of these regions. However, the possible existence of RNA transcripts having only small regions of homology to our probes or RNA transcripts from other regions not represented in our probes cannot be excluded.

In summary, the evolution of the oncogenic virus REV-T from $c$-rel involved many steps. From the experiments described here, the minimum events required to generate $v$-rel from $c$-rel involved recombination between helper virus and $c$-rel, splicing of apparent large introns, perhaps analogous to splicing of $\alpha$-globin introns in retrovirus construction (25a), base pair mutations, and deletion of at least part of an exon from c-rel.

\section{ACKNOWLEDGMENTS}

We thank Virginia Goiffon, Susan Hellenbrand, and Hwey Yang for excellent technical assistance and M. Emerman, J. Mertz, C. Miller, R. Risser, T. Schedl, and B. Sugden for helpful comments on the manuscript.

This investigation was supported by Public Health Service research grants CA-07175 and CA-22443 from the National Cancer Institute. K.C.W. was supported by Public Health Service training grant CA-09135 from the National Cancer Institute. H.M.T. is an American Cancer Society Research Professor.

\section{ADDENDUM IN PROOF}

Since the submission of this manuscript, we have learned that the 1.9-kbp EcoRI fragment detected in genomic chicken DNA sequences with the $5^{\prime} v$-rel probe (see Fig. 1) also hybridizes to a $c$-rel HindIII/ HindIII fragment (1.7 to $2.4 \mathrm{kbp}$ in Fig. 2). This result indicates that a portion of $c$-rel is duplicated in the chicken genome. Furthermore, we have learned that the BgIIII to EcoRI portion of $\lambda 1059 \mathrm{c}$-rel $5(-1.0$ to $1.0 \mathrm{kbp}$ in the $c$-rel map, Fig. 2) does not represent a portion of the 1.9-kbp EcoRI fragment detected in genomic chicken sequences. We believe these results indicate that there is a second locus in chicken DNA with a partial duplication of the $c$-rel sequences.

\section{LITERATURE CITED}

1. Abelson, H. T., and L. S. Rabstein. 1970. Influence of prednisolone on Moloney leukemogenic virus in BALB/c mice. Cancer Res. 30:2208-2212.

2. Aviv, H., and P. Leder. 1972. Purification of biologically active globin messenger RNA by chromatography on oligothymidylic acid-cellulose. Proc. Natl. Acad. Sci. U.S.A. 69:1408-1412.

3. Baltimore, D., J. Wang, S. Goff, and C. Queen. 1982.
Origin, structure and function of the transforming region of the Abelson murine leukemia virus. J. Cell. Biochem. (Suppl.) 6:189.

4. Bishop, J. M. 1981. Enemies within: the genesis of retrovirus oncogenes. Cell 23:5-6.

5. Blattner, F. R., B. G. Williams, A. E. Blechl, K. Denniston-Thompson, H. E. Faber, L. A. Furlong, D. J. Grunwald, D. O. Kiefer, D. O. Moore, J. W. Schumm, E. I. Sheldon, and O. Smithies. 1977. Charon phages: safer derivatives of bacteriophage lambda for DNA cloning. Science 196:161-169.

6. Breitman, M. L., M. M. C. Lai, and P. K. Vogt. 1980. The genomic RNA of avian reticuloendotheliosis virus REV. Virology 100:450-461.

7. Chen, I.S.Y., T. W. Mak, J. J. O'Rear, and H. M. Temin. 1981. Characterization of reticuloendotheliosis virus strain T DNA and isolation of a novel variant of reticuloendotheliosis virus strain $\mathrm{T}$ by molecular cloning. $\mathrm{J}$. Virol. 40:800-811.

7a.Chen, I. S. Y., and H. M. Temin. 1982. Substitution of $5^{\prime}$ helper virus sequences into non-rel portion of reticuloendotheliosis virus strain $T$ suppresses transformation of chicken spleen cells. Cell 31:111-120.

8. Cofin, J. M. 1982. Structure of the retroviral genome, p. 261-368. In R. Weiss, N. Teich, H. Varmus, and J. Coffin (ed.), The molecular biology of RNA tumor viruses, 2nd ed. RNA tumor viruses. Cold Spring Harbor Laboratory, Cold Spring Harbor, N.Y.

9. Dalla Favera, R., E. P. Gelmann, R. C. Gallo, and F. Wong-Staal. 1981. A human onc gene homologous to the transforming gene ( $\mathrm{v}$-sis) of simian sarcoma virus. Nature (London) 292:31-35.

10. Ellis, R. W., D. DeFeo, T. Y. Shih, M. A. Gonda, H. A. Young, N. Tsuchida, D. R. Lowy, and E. M. Scolnick. 1981. The p21 src genes of Harvey and Kirsten sarcoma viruses originate from divergent members of a family of normal vertebrate genes. Nature (London) 292:506-511.

11. Franchini, G., J. Even, C. J. Sherr, and F. Wong-Staal. 1981. onc sequences ( $v-f e s)$ of Snyder-Theilen feline sarcoma virus are derived from noncontiguous regions of a cat cellular gene (c-fes). Nature (London) 290:154-157.

12. Fritsch, E., and H. M. Temin. 1977. Formation and structure of infectious DNA of spleen necrosis virus. J. Virol. 21:119-130.

13. Gonda, M. A., N. R. Rice, and R. V. Gilden. 1980. Avian reticuloendotheliosis virus: characterization of the highmolecular-weight viral RNA in transforming and helper virus populations. J. Virol. 34:743-751.

14. Hoelzer, J. D., R. B. Lewis, C. R. Wasmuth, and H. R. Bose. 1980. Hematopoietic cell transformation by avian reticuloendotheliosis: characterization of the genetic defect. Virology 100:462-474.

15. Holmes, D. S., and J. Bonner. 1973. Preparation, molecular weight, base composition and secondary structure of giant nuclear ribonucleic acid. Biochemistry 12:23302338.

16. Karn, J., S. Brenner, L. Barnett, and G. Cesareni. 1980. Novel bacteriophage $\lambda$ cloning vector. Proc. Natl. Acad. Sci. U.S.A. 77:5172-5176.

17. Nunberg, J. H., R. J. Kaufman, A. C. Y. Chang, S. N. Cohen, and R. T. Schimke. 1980. Structure and genomic organization of the mouse dihydrorolate reductase gene. Cell 19:355-364.

18. Oskarsson, M., W. L. McClements, D. G. Blair, J. V. Maizel, and G. F. Vande Woude. 1980. Properties of a normal mouse cell DNA sequence (sarc) homologous to the src sequence of Moloney sarcoma virus. Science 207:1222-1224.

19. Parker, R. C., H. E. Varmus, and J. M. Bishop. 1981. Cellular homologue (c-src) of the transforming gene of Rous sarcoma virus: isolation, mapping, and transcriptional analysis of c-src and flanking regions. Proc. Natl Acad. Sci. U.S.A. 78:5842-5846.

20. Perbal, B., and M. A. Baluda. 1982. Avian myeloblastosis virus transforming gene is related to unique chicken DNA 
regions separated by at least one intervening sequence. J. Virol. 41:250-257.

21. Robins, T., K. Bister, C. Garon, T. Papas, and P. Duesberg. 1982. Structural relationship between a normal chicken DNA locus and the transforming gene of the avian acute leukemia virus MC29. J. Virol. 41:635-642.

22. Ross, J. 1976. A precursor of globin messenger RNA. J. Mol. Biol. 106:403-420.

23. Shalloway, D., A. D. Zelenetz, and G. M. Cooper. 1981. Molecular cloning and characterization of the chicken gene homologous to the transforming gene of Rous sarcoma virus. Cell 24:531-541.

24. Shimotohno, K., S. Mizutani, and H. M. Temin. 1980. Sequence of retrovirus provirus resembles that of bacterial transposable elements. Nature (London) 285:550-554.

25. Shimotohno, K., and H. M. Temin. 1981. Formation of infectious progeny virus after insertion of herpes simplex thymidine kinase gene into DNA of an avian retrovirus. Cell 26:67-77.

25a.Shimotohno, K., and H. M. Temin. 1982. Loss of interven- ing sequences in genomic mouse $\alpha$-globin DNA inserted in an infectious retrovirus vector. Nature (London) 299:265268.

27. Theilen, G. H., R. F. Zeigel, and M. J. Twiehaus. 1966. Biological studies with RE virus (strain $T$ ) that induces reticuloendotheliosis in turkeys, chicken, and Japanese quail. J. Natl. Cancer Inst. 37:731-743.

28. Thomas, P. S. 1980 . Hybridization of denatured RNA and small DNA fragments transferred to nitrocellulose. Proc. Natl. Acad. Sci. U.S.A. 77:5201-5205.

29. Vennstrom, B., and J. M. Bishop. 1982. Isolation and characterization of chicken DNA homologous to the two putative oncogenes of avian erythroblastosis virus. Cell 28:135-143.

30. Witter, R. L. 1979. A proposed method for designating avian cell lines and transplantable tumours. Avian Pathol. $8: 487-498$.

31. Wong, T. C., and M. M. C. Lai. 1981. Avian reticuloendotheliosis virus contains a new class of oncogene of turkey origin. Virology 111:289-293. 